\title{
Friction term discretization and limitation to preserve stability and conservation in the 1D shallow-water model: application to unsteady irrigation and river flow.
}

\author{
J. Burguete ${ }^{1, *}$, P. García-Navarro² and J. Murillo \\ ${ }^{1}$ Suelo y Agua. Estación Experimental Aula Dei. CSIC. Zaragoza. Spain \\ 2 Fluid Mechanics. CPS. University of Zaragoza. Zaragoza. Spain
}

\section{SUMMARY}

Friction is one of the relevant forces included in the momentum equation of the one-dimensional shallow-water model. This work shows that a pointwise discretization of the friction term unbalances this term with the rest of the terms in the equation in steady state. On the other hand, an upwind discretization of the friction term ensures the correct discrete balance. Furthermore, a conservative technique based on the limitation of the friction value is proposed in order to avoid unbounded values of the friction term in unsteady cases of advancing front over dry and rough surfaces. This limitation improves the quality of unsteady solutions in wet/dry fronts and guarantees the numerical stability in cases with dominant friction terms. The proposed discretization is validated in some test cases with analytical solution or with measured data and used in some practical cases. Copyright (c) 2007 John Wiley \& Sons, Ltd.

KEY WORDS: Shallow water, Source term, Flow resistance, Roughness, Numerical models, Irrigation,

*Correspondence to: jburguete@eead.csic.es 
River flow, Numerical stability

\section{INTRODUCTION}

The one-dimensional shallow-water model involves cross-sectional averaging of the original flow equations. One of the main consequences of such cross sectional averaging is that the viscous flow boundary condition at solid walls and bottom is transformed into a drag/friction force source term. The form of this friction force term is closely related to the assumed velocity profile and therefore to the underlying turbulence model. To model friction, empirical or semiempirical methods have traditionally been applied $[1,2,3]$. They are all dependent on the surface roughness as well as on the flow characteristics, and their coefficients can be found in tabular form in many references as, for instance, [4]. Several authors have reported theoretical studies devoted to model the friction force based on velocity profiles $[5,6]$.

The main objective of the present work is related to the study of the best way of incorporating the friction forces into numerical simulation models of steady and unsteady flow and to the evaluation of the interaction between a dominant friction term in the equations and the stability constraints within a model at the discrete level. Few previous works have treated in detail the consequences of the discretization of the friction term. The most commonly reported procedure $[7,8]$ is the pointwise discretization of the term independently of the methodology used for the rest of the system, for simplicity reasons. A unified discretization of all the source terms was suggested previously $[9,10]$ over the basis of the widely reported convenience of using an upwind discretization for the bed slope source terms in the context of upwind schemes $[11,12]$. The unified discretization also applied to the friction source terms is considered in detail in 
this work and the ability to produce conservative solutions is evaluated in a case of steady open channel flow with analytical solution.

Numerical stability constraints of explicit schemes for the 1D shallow water equations are basically derived from the homogeneous system of equations, leading to the well-known Courant-Friedrichs-Lewy (CFL) condition [13]. It has been previously reported that this constraint over the time step is not enough to guarantee stability in cases of high relative roughness, that is, in presence of important roughness values and low water depth. The usual cure is to reduce the time step over the minimum value given by the CFL condition or to apply an implicit treatment of the pointwise discretized friction source term. A new stability condition related to the friction term and leading to a new requirement on the spatial grid size was derived in [6] based on the physical idea that, in front advance over dry bed, friction can, at most, stop the water front movement but never produce a change of sign in the water velocity. That approach is correct but can result in excessively fine grids to achieve stability, hence resulting in inefficient calculations. Following that line of reasoning, it can also be considered that the maximum allowable value of the friction force is that able to prevent the water movement. This can be considered an obvious physical condition but present day models of the friction term usually violate this principle at the discrete level. In this work, we shall explore a numerical limitation of the friction value in order to bound within physical limits the friction force making sure, at the same time, that the scheme remains conservative, stable and efficient.

Finally, the optimal discretization of the friction term will be inserted in a second order TVD scheme and this will be applied to practical cases. First, a dam break flow problem in a reach of a mountain river and second to a couple of test cases of unsteady flow with experimental 
data, one dealing with a tsunami propagation physical model and the other dealing with the advance of a surface irrigation flow.

\section{BASIC EQUATIONS}

1D open channel flow can be modelled using the Saint-Venant equations [14]. The conservative form of these equations can be expressed in vector form as:

$$
\frac{\partial \mathbf{u}}{\partial t}+\frac{\partial \mathbf{F}^{c}}{\partial x}=\mathbf{H}^{c}
$$

with $\mathbf{u}$ the conserved variable, and $\mathbf{F}^{c}$ and $\mathbf{H}^{c}$ the conservative flux and source terms respectively:

$$
\mathbf{u}=\left(\begin{array}{c}
A \\
Q
\end{array}\right), \quad \mathbf{F}^{c}=\left(\begin{array}{c}
Q \\
\frac{\beta Q^{2}}{A}+g I_{1}
\end{array}\right), \quad \mathbf{H}^{c}=\left(\begin{array}{c}
0 \\
g\left(I_{2}+A S_{0}\right)-T
\end{array}\right)
$$

where $A$ is the wetted cross section, $Q$ the discharge, $g$ the gravitational acceleration, $S_{0}$ the main bed slope, $T$ the friction stress over the solid surface in the channel reach, $\beta$ is a coefficient that appears as a result of the assumption of variable velocity in the cross section:

$$
\beta=\frac{A}{Q^{2}} \int_{A} v_{x}^{2} d A
$$

with $v_{x}=v_{x}(x, y, z, t)$ the $x$ component of the local point flow velocity, and $I_{1}, I_{2}$ pressure forces:

$$
I_{1}=\int_{0}^{H} \sigma\left(x, z^{\prime \prime}\right)\left(H-z^{\prime \prime}\right) d z^{\prime \prime}, \quad I_{2}=\int_{0}^{H} \frac{\partial \sigma\left(x, z^{\prime \prime}\right)}{\partial x}\left(H-z^{\prime \prime}\right) d z^{\prime \prime}
$$

with $H$ the maximum water depth at the cross section and $\sigma$ the section width at a level $z^{\prime \prime}$ with reference to the minimum bottom level $z_{b}$ (see Fig. 1). 


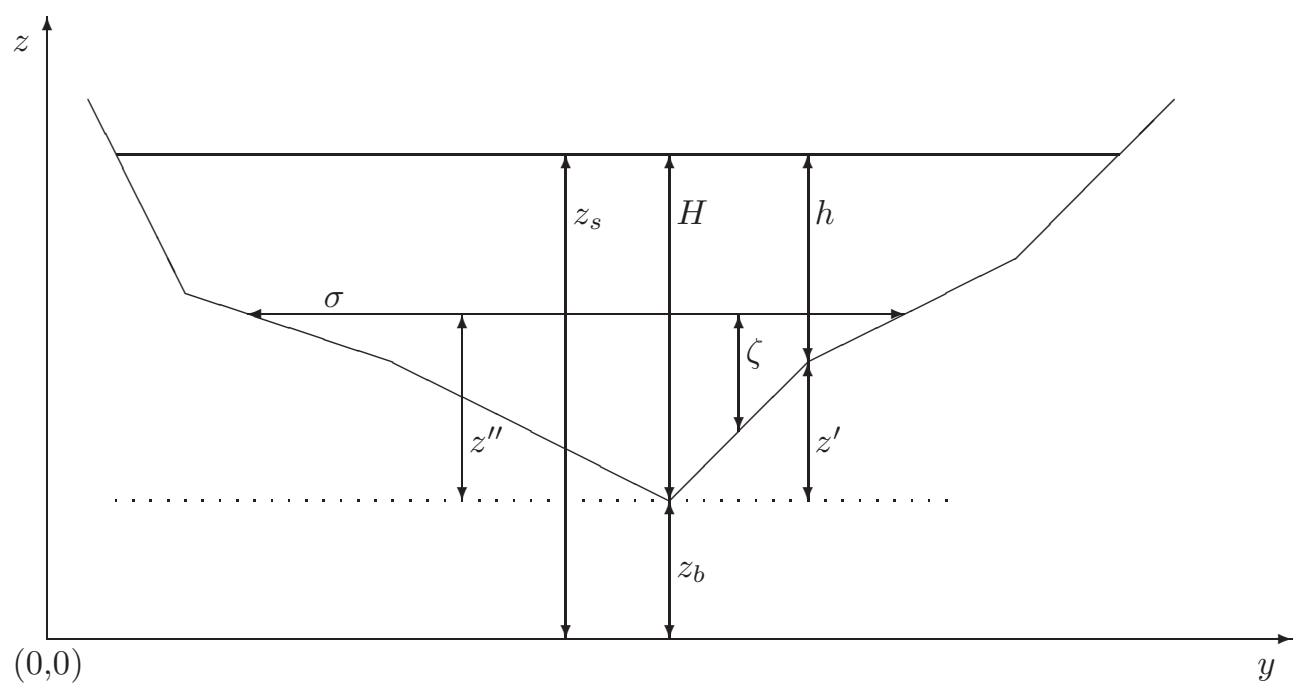

Figure 1. Coordinate system in a cross section.

The flux Jacobian is:

$$
\mathbf{J}=\frac{\partial \mathbf{F}^{c}}{\partial \mathbf{u}}=\left(\begin{array}{cc}
0 & 1 \\
c^{2}-\beta u^{2} & 2 \beta u
\end{array}\right)
$$

with $u=Q / A$ the average flow velocity and $c=\sqrt{g A / B}$ the celerity of the infinitesimal waves, where $B$ is the cross sectional top width. The eigenvalues of this Jacobian are:

$$
\lambda_{1}=\beta u+\sqrt{\left(\beta^{2}-\beta\right) u^{2}+c^{2}}, \quad \lambda_{2}=\beta u-\sqrt{\left(\beta^{2}-\beta\right) u^{2}+c^{2}}
$$

The matrices that make diagonal this Jacobian are:

$$
\mathbf{P}=\left(\begin{array}{cc}
1 & 1 \\
\lambda_{1} & \lambda_{2}
\end{array}\right), \quad \boldsymbol{\Lambda}=\left(\begin{array}{cc}
\lambda_{1} & 0 \\
0 & \lambda_{2}
\end{array}\right), \quad \mathbf{J}=\mathbf{P} \boldsymbol{\Lambda} \mathbf{P}^{-1}
$$

The Jacobian of the source term is:

$$
\mathbf{K}=\frac{\partial \mathbf{H}^{c}}{\partial \mathbf{u}}=\left(\begin{array}{cc}
0 & 0 \\
-g\left(\frac{\partial z_{s}}{\partial x}-\frac{1}{B} \frac{\partial A}{\partial x}\right)-\frac{\partial T}{\partial A} & -\frac{\partial T}{\partial Q}
\end{array}\right)
$$

with $z_{s}$ the water surface level. 
A last, simple and very convenient form of the equations is the quasi-conservative form.

Taking into account that:

$$
\frac{\partial I_{1}}{\partial x}=I_{2}+A \frac{\partial z_{s}}{\partial x}
$$

and inserting in (2):

$$
\frac{\partial \mathbf{u}}{\partial t}+\frac{\partial \mathbf{F}^{q c}}{\partial x}=\mathbf{H}^{q c}
$$

with $\mathbf{F}^{q c}$ and $\mathbf{H}^{q c}$ the quasi-conservative flux and source terms:

$$
\mathbf{F}^{q c}=\left(\begin{array}{c}
Q \\
\frac{\beta Q^{2}}{A}
\end{array}\right), \quad \mathbf{H}^{q c}=\left(\begin{array}{c}
0 \\
-g A \frac{\partial z_{s}}{\partial x}-T
\end{array}\right)
$$

\section{FRICTION MODELS}

\subsection{Gauckler-Manning friction model}

In the empirical Gauckler-Manning model, the friction slope $S_{f}$ can be expressed as $[2,3]$ :

$$
S_{f}=\frac{T}{g A}=\frac{n^{2}|Q| Q}{R^{4 / 3}}
$$

with $R=A / P$ the hydraulic radius, $P$ the wetted perimeter and $n$ the Gauckler-Manning coefficient. This model is only valid in cases of uniform flow velocity distribution in a cross section. A kinematic friction stress $\tau$ at a point in this model can be defined as:

$$
\tau d P=g h S_{f} d y=g n^{2}|U| U h^{-1 / 3} d y
$$

with $h=H-z^{\prime}$ the local water depth, $z^{\prime}$ the local bottom level with respect to the minimum bottom level in the cross section (see Figure 1), $U$ the depth-averaged water velocity and $y$ the transversal coordinate. In irregular sections or compound channels, the velocity cannot be considered uniform in a cross section. In these cases a constant slope model can be used [6]. 
Working out the averaged velocity from the last expression:

$$
U=\frac{ \pm \sqrt{\left|S_{f}\right|} h^{2 / 3}}{n}
$$

Furthermore, if the friction slope is constant in a section:

$$
Q=\int_{A} U h d y= \pm \sqrt{\left|S_{f}\right|} \int_{A} \frac{h^{5 / 3}}{n} d y
$$

Then:

$$
S_{f}=\frac{|Q| Q}{\left(\int_{A} \frac{h^{5 / 3}}{n} d y\right)^{2}}
$$

This model also enables an estimate of the cross sectional momentum distribution $\beta$ parameter:

$$
\beta=\frac{A \int_{A} U^{2} h d y}{Q^{2}}=\frac{A S_{f}}{|Q| Q} \int_{A} \frac{h^{7 / 3}}{n^{2}} d y
$$

\subsection{Power law velocity model}

A power law velocity model was proposed in [6] where it proved more accurate than the Gauckler-Manning model in high relative roughness situations. This model assumes that the bed irregularities are of average size $l$ and that, in smooth channels, $l$ can be identified with the laminar sublayer thickness. Furthermore, this model assumes that the velocity profile can be fit by means of a power function in the roughness upper zone, being negligible in the lower zone:

$$
v_{x}(x, y, z, t)=u_{l}(x, y, t)\left(\frac{\zeta}{l}\right)^{b}, \text { if } \zeta \geq l
$$

where $\zeta=z-z_{b}-z^{\prime}$ is the vertical distance to the bottom level, $b$ is a fitting exponent and $u_{l}(x, y, t)$ is the water velocity at a distance $l$ of the bed, as represented in Fig. 2. Integration in the vertical coordinate leads to:

$$
h U=\int_{0}^{h} v_{x} d \zeta=\frac{u_{l}}{b+1}\left(\frac{h^{1+b}}{l^{b}}-l\right)
$$




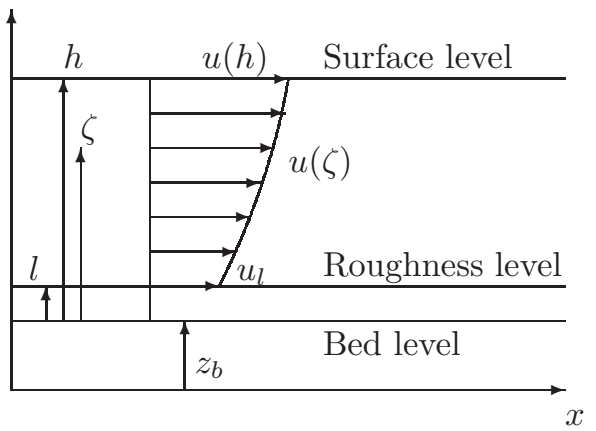

Figure 2. Power function fitting the velocity profile.

If the flow can be considered fully turbulent, as it happens in most channel and river flow situations, the friction stress can be expressed as [6]:

$$
\tau d P \approx \epsilon\left|u_{l}\right| u_{l} d y=\frac{\epsilon(b+1)^{2}|U| U}{\left[\left(\frac{h}{l}\right)^{b}-\frac{l}{h}\right]^{2}} d y, \text { if } h>l
$$

with $\epsilon$ a dimensionless aerodynamic constant depending on the roughness characteristics and on the Reynolds number. This friction law is only valid for $h>l$. If $h<l$ a zero velocity condition is imposed for numerical stabilisation of the advance over dry bed. Working out the friction slope:

$$
S_{f}=\frac{\tau}{g h} \frac{d P}{d h}=\frac{\epsilon(b+1)^{2}|U| U}{g\left(\frac{h^{b+(1 / 2)}}{l^{b}}-\frac{l}{\sqrt{h}}\right)^{2}}
$$

hence, the velocity:

$$
U= \pm \sqrt{\frac{g\left|S_{f}\right|}{\epsilon}} \frac{1}{b+1}\left(\frac{h^{b+(1 / 2)}}{l^{b}}-\frac{l}{\sqrt{h}}\right)
$$

so that, applying the constant friction slope hypothesis:

$$
Q=\int_{A} U h d y= \pm \sqrt{g\left|S_{f}\right|} \int_{A} \frac{1}{(b+1) \sqrt{\epsilon}}\left(\frac{h^{b+(3 / 2)}}{l^{b}}-l \sqrt{h}\right) d y
$$


and we get for $S_{f}$ :

$$
S_{f}=\frac{|Q| Q}{g\left[\int_{A} \frac{1}{(b+1) \sqrt{\epsilon}}\left(\frac{h^{b+(3 / 2)}}{l^{b}}-l \sqrt{h}\right) d y\right]^{2}}
$$

This model also enables an estimate of the $\beta$ parameter. Using (3), (18), (19) and (22):

$$
\beta=\frac{g A S_{f}}{|Q| Q} \int_{A} \frac{1}{(2 b+1) \epsilon}\left(\frac{h^{2 b+2}}{l^{2 b}}-l h\right) d y
$$

\section{CONSERVATIVE NUMERICAL SCHEMES}

\subsection{Unified discretization of the friction term}

The conservative form of the system of differential equations (1) can be solved by means of a conservative scheme. The following vector is defined for convenience [10]:

$$
\mathbf{G}_{i+(1 / 2)}=\left(\mathbf{H}^{q c}-\frac{\delta \mathbf{F}^{q c}}{\delta x}\right)_{i+(1 / 2)}
$$

The unified discretization of the friction term consists of a similar numerical treatment of the flux, friction and other source terms in the equation. A general conservative scheme with unified discretization can be expressed as $[9,10,15]$ :

$$
\left(1-\theta \Delta t \mathbf{K}_{i}^{n}\right) \Delta \mathbf{u}_{i}^{n}=\Delta t\left(\mathbf{G}_{i-(1 / 2)}^{L}+\mathbf{G}_{i+(1 / 2)}^{R}\right)
$$

where $\mathbf{K}$ is the source term Jacobian, $\theta$ is a coefficient controlling the implicitness of the source term and $\mathbf{G}^{L, R}$ represents the wave discretization particular to each numerical scheme.

We shall define the upwind matrices as:

$$
\boldsymbol{\Omega}^{ \pm}=\frac{1}{2} \mathbf{P}\left(\begin{array}{cc}
1 \pm \operatorname{sign}\left(\lambda_{1}\right) & 0 \\
0 & 1 \pm \operatorname{sign}\left(\lambda_{2}\right)
\end{array}\right) \mathbf{P}^{-1}
$$


and the transcritical artificial viscosity coefficient as [15]:

$$
\nu_{i+(1 / 2)}^{n}=\max _{k}\left\{\begin{array}{cl}
\frac{1}{4}\left[\delta\left(\lambda_{k}\right)-2\left|\lambda_{k}\right|\right]_{i+(1 / 2)}, & \text { if }\left(\lambda_{k}\right)_{i}^{n}<0 \text { and }\left(\lambda_{k}\right)_{i+1}^{n}>0 \\
0, & \text { otherwise }
\end{array}\right.
$$

The second order vectors are defined as [16]:

$$
\mathbf{L}^{ \pm}=\left(\mathbf{1} \mp \boldsymbol{\Lambda}^{ \pm} \frac{\Delta t}{\delta x}\right) \mathbf{P}^{-1} \mathbf{G}^{ \pm}
$$

and the flux limiting matrices as:

$$
\Psi_{i+(1 / 2)}^{ \pm}=\left(\begin{array}{ccc}
\Psi\left(\frac{\left(\mathbf{L}^{ \pm}\right)_{i+(1 / 2) \pm 1}^{1}}{\left(\mathbf{L}^{ \pm}\right)_{i+(1 / 2)}^{1}}\right) & 0 & 0 \\
0 & \Psi\left(\frac{\left(\mathbf{L}^{ \pm}\right)_{i+(1 / 2) \pm 1}^{2}}{\left(\mathbf{L}^{ \pm}\right)_{i+(1 / 2)}^{2}}\right) & 0 \\
0 & 0 & \Psi\left(\frac{\left(\mathbf{L}^{ \pm}\right)_{i+(1 / 2) \pm 1}^{3}}{\left(\mathbf{L}^{ \pm}\right)_{i+(1 / 2)}^{3}}\right)
\end{array}\right)
$$

where $\mathbf{L}^{k}$ represents the $k$ component of the vector $\mathbf{L}$ and $\Psi$ is the flux limiter function. Many particular flux limiter functions are defined in previous works $[17,18,19]$. We use:

- "Superbee" [17]: $\Psi(r)=\max [0, \min (1,2 r), \min (2, r)]$

Then, the wave decomposition of the second order TVD scheme is [16]:

$$
\begin{aligned}
\mathbf{G}_{i+(1 / 2)}^{L} & =\left(\mathbf{G}^{+}-\nu \frac{\delta \mathbf{u}}{\delta x}\right)_{i+(1 / 2)}^{n}-\frac{1}{2}\left(\mathbf{P} \boldsymbol{\Psi}^{+} \mathbf{L}^{+}\right)_{i-(1 / 2)}^{n}+\frac{1}{2}\left(\mathbf{P} \mathbf{\Psi}^{-} \mathbf{L}^{-}\right)_{i+(3 / 2)}^{n}, \\
\mathbf{G}_{i+(1 / 2)}^{R} & =\left(\mathbf{G}^{-}+\nu \frac{\delta \mathbf{u}}{\delta x}\right)_{i+(1 / 2)}^{n}+\frac{1}{2}\left(\mathbf{P} \Psi^{+} \mathbf{L}^{+}\right)_{i-(1 / 2)}^{n}-\frac{1}{2}\left(\mathbf{P} \Psi^{-} \mathbf{L}^{-}\right)_{i+(3 / 2)}^{n}
\end{aligned}
$$

\subsection{Pointwise discretization}

Due to the form of the friction source term, it is common to find applications using a pointwise discretization. In that case, the quasi-conservative source term (10) is split in two parts, one of them involving only the friction term, that are treated separately:

$$
\mathbf{H}^{q c}=\mathbf{R}+\mathbf{S}
$$


with

$$
\mathbf{R}=\left(\begin{array}{c}
0 \\
-T
\end{array}\right), \quad \mathbf{S}=\left(\begin{array}{c}
0 \\
-g A \frac{\partial z_{s}}{\partial x}
\end{array}\right)
$$

Redefining now the vector:

$$
\mathbf{G}_{i+(1 / 2)}=\left(\mathbf{S}-\frac{\delta \mathbf{F}^{q c}}{\delta x}\right)_{i+(1 / 2)}
$$

A conservative scheme, like the one defined in the above subsection, is then applied to the flux derivative and bed slope source term so that the friction term is later added with fully implicit discretization as:

$$
\Delta \mathbf{u}_{i}^{n}=\Delta t\left(\mathbf{R}_{i}^{n+1}+\mathbf{G}_{i-(1 / 2)}^{L}+\mathbf{G}_{i+(1 / 2)}^{R}\right)
$$

Even though this discretization is widespread and recommended in several works $[7,8]$ due to its simplicity and the possibility to use a fully implicit discretization of the friction term, figure 5 shows that it produces the unbalance of the friction term and the rest of the terms in the equation in steady state.

\section{NUMERICAL STABILITY AND LIMITATION OF THE FRICTION TERM}

Explicit numerical schemes applied to solve the unsteady Saint-Venant equations are traditionally said to be numerically stable if the time step and grid size chosen are linked by the CFL condition [13] based on the advective part of the equations. The implementation of this condition in equation (1), with the flux Jacobian eigenvalues (6), gives:

$$
\mathrm{CFL}=\Delta t \frac{\beta|u|+\sqrt{\left(\beta^{2}-\beta\right) u^{2}+c^{2}}}{\delta x} \leq 1
$$

with CFL the dimensionless Courant-Friedrichs-Lewy number.

The friction source term in the Saint-Venant (1) equations is often one of the dominant terms, especially in river and overland flow or in surface irrigation applications. This relevance 
has consequences at the discrete level particularly as far as numerical stability is concerned and it is essential to establish a stability conditions that takes them into account. Assuming a domain as sketched in Figure 3, a flat surface level develops over a rough adverse slope.

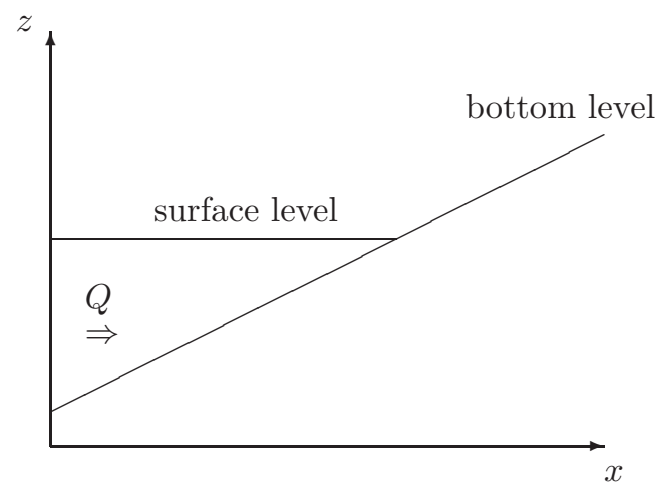

Figure 3. Rough prismatic channel with adverse slope and flat surface level.

Let us call $i$ the dry grid node immediately next to the wet/dry front. In this situation, the following properties hold and are expected:

$$
Q_{i}^{n}=A_{i}^{n}=0, \quad Q_{i-1}^{n} \geq 0, \quad A_{i-1}^{n} \geq 0, \quad Q_{i}^{n+1} \geq 0, \quad A_{i}^{n+1} \geq 0
$$

The following can be written for an explicit conservative numerical scheme with unified discretization of the friction term applied to the dynamic equation at node $i$ :

$$
Q_{i}^{n+1}=Q_{i}^{n}-\Delta t\left[T+\frac{\delta}{\delta x}\left(\frac{Q^{2}}{A}\right)\right]_{i-(1 / 2)}^{L}=\frac{\Delta t}{\delta x}\left[-\frac{T \delta x}{2}+\frac{Q^{2}}{A}\right]_{i-1}^{n}
$$

where the arithmetic mean has been performed $\left(T_{i-(1 / 2)}=\left(T_{i-1}+T_{i}\right) / 2\right)$. In order to enforce the physical conditions (38), the explicit numerical scheme must satisfy:

$$
\delta x \leq \frac{2 Q^{2}}{A|T|}=\frac{2 R^{4 / 3}}{g n^{2}}
$$

This condition was proposed in [6] where the usefulness of the restriction was proved in a practical case. 
It is important to note that the additional stability condition (40) is not necessary when a fully implicit pointwise separate discretization of the friction term is used. Applying (36) to the momentum conservation equation:

$$
Q_{i}^{n+1}=Q_{i}^{n}-\Delta t\left(\frac{g n^{2}|Q| Q P^{\frac{4}{3}}}{A^{\frac{7}{3}}}\right)_{i}^{n+1}-\Delta t \frac{\delta}{\delta x}\left(\frac{Q^{2}}{A}\right)_{i-(1 / 2)}^{L}-\Delta t \frac{\delta}{\delta x}\left(\frac{Q^{2}}{A}\right)_{i-(1 / 2)}^{R}
$$

leading to:

$$
\left(1+\Delta t \frac{g n^{2}|Q| P^{\frac{4}{3}}}{A^{\frac{7}{3}}}\right)_{i}^{n+1} Q_{i}^{n+1}=Q_{i}^{n}-\Delta t \frac{\delta}{\delta x}\left(\frac{Q^{2}}{A}\right)_{i-(1 / 2)}^{L}-\Delta t \frac{\delta}{\delta x}\left(\frac{Q^{2}}{A}\right)_{i-(1 / 2)}^{R}
$$

using, for instance, a centred numerical scheme:

$$
\left(1+\Delta t \frac{g n^{2}|Q| P^{\frac{4}{3}}}{A^{\frac{7}{3}}}\right)_{i}^{n+1} Q_{i}^{n+1}=Q_{i}^{n}-\frac{\Delta t}{2}\left[\frac{\delta}{\delta x}\left(\frac{Q^{2}}{A}\right)_{i+(1 / 2)}^{n}+\frac{\delta}{\delta x}\left(\frac{Q^{2}}{A}\right)_{i-(1 / 2)}^{n}\right]
$$

and, at the wet/dry front, the following can be written:

$$
Q_{i}^{n+1}=\frac{\Delta t}{2 \Delta x} \frac{\left(\frac{Q^{2}}{A}\right)_{i+1}^{n}}{\left(1+\Delta t \frac{g n^{2}|Q| P^{\frac{4}{3}}}{A^{\frac{7}{3}}}\right)_{i}^{n+1}} \geq 0
$$

that meets the stability condition (38). This superior stability of the fully implicit pointwise discretization of the friction term, besides the simplicity of its formulation, justifies the widespread application $[7,8]$ although figure 5 shows that it produces the unbalance of the friction term and the rest of the terms in the equation in steady state.

From the physical point of view the friction force has an upper bound that cannot be exceeded: the maximum value able to stop the flow. This fact, evident at the physical level, can be violated at the discrete level and this is reason why friction terms produce numerical instability in the solution. Then a numerical scheme subject only to the CFL condition cannot automatically satisfy conditions (38), becoming necessary the additional restriction (40) with the unified discretization of the friction term. 
In this work, a different point of view to avoid the numerical instability associated to the friction term is proposed. It consists of the suitable limitation of the numerical friction force value. Integration of the quasi-conservative equation of the momentum conservation (10) in a grid cell and in a time step:

$$
\begin{aligned}
\int_{t^{n}}^{t^{n+1}} d t \int_{x_{i}}^{x_{i+1}} d x\left[\frac{\partial Q}{\partial t}\right. & \left.+\frac{\partial}{\partial x}\left(\frac{\beta Q^{2}}{A}\right)\right]=\int_{x_{i}}^{x_{i+1}} d x \Delta Q^{n}+\int_{t^{n}}^{t^{n+1}} d t \frac{\delta}{\delta x}\left(\frac{\beta Q^{2}}{A}\right)_{i+(1 / 2)}= \\
& =-\int_{t^{n}}^{t^{n+1}} d t \int_{x_{i}}^{x_{i+1}} d x\left(g A \frac{\partial z_{s}}{\partial x}+T\right)
\end{aligned}
$$

so that, making an explicit discretization:

$$
\Delta Q_{i+(1 / 2)}^{n}=-\Delta t\left[\frac{\delta}{\delta x}\left(\frac{\beta Q^{2}}{A}\right)_{i+(1 / 2)}^{n}+\left(g A \frac{\delta z_{s}}{\delta x}\right)_{i+(1 / 2)}^{n}+T_{i+(1 / 2)}^{n}\right]
$$

where arithmetical average has been chosen $Q_{i+(1 / 2)}=\left(Q_{i+1}+Q_{i}\right) / 2$. We shall define:

$$
Q_{i+(1 / 2)}^{*}=Q_{i+(1 / 2)}^{n}-\Delta t\left[\frac{\delta}{\delta x}\left(\frac{\beta Q^{2}}{A}\right)_{i+(1 / 2)}^{n}+\left(g A \frac{\delta z_{s}}{\delta x}\right)_{i+(1 / 2)}^{n}\right]
$$

involving all the forces except friction forces. Given that the maximum effect of the friction force is to stop the water flow, a necessary condition in the solution is that the updated value of the discharge at a point $Q^{n+1}$ after the addition of the discrete friction term retains the same sign of the value at the previous time level $Q^{*}$, that is:

$$
Q_{i+(1 / 2)}^{n+1} Q_{i+(1 / 2)}^{*} \geq 0 \Rightarrow\left(Q_{i+(1 / 2)}^{*}-\Delta t T_{i+(1 / 2)}^{n}\right) Q_{i+(1 / 2)}^{*} \geq 0
$$

providing a numerical bounding value for the allowable friction force:

$$
|T|_{i+(1 / 2)}^{n} \leq \frac{|Q|_{i+(1 / 2)}^{*}}{\Delta t}
$$

When the numerical friction force exceeds this value, it will be limited to the maximum value. It is important to note that the limitation is automatically hold in steady cases. In this case $\Delta Q^{n}=0$ and using (46):

$$
T_{i+(1 / 2)}^{n}=-\frac{\delta}{\delta x}\left(\frac{\beta Q^{2}}{A}\right)_{i+(1 / 2)}^{n}-\left(g A \frac{\delta z_{s}}{\delta x}\right)_{i+(1 / 2)}^{n}
$$


Inserting in (47):

$$
Q_{i+(1 / 2)}^{*}=Q_{i+(1 / 2)}^{n}+\Delta t T_{i+(1 / 2)}^{n}
$$

and using that, by definition, $T$ has the same $\operatorname{sign}$ as $Q$ it is true that (49):

$$
\frac{|Q|_{i+(1 / 2)}^{*}}{\Delta t}=\frac{\left|Q_{i+(1 / 2)}^{n}+\Delta t T_{i+(1 / 2)}^{n}\right|}{\Delta t}=\frac{|Q|_{i+(1 / 2)}^{n}}{\Delta t}+|T|_{i+(1 / 2)}^{n} \geq|T|_{i+(1 / 2)}^{n}
$$

\section{APPLICATIONS}

\subsection{Hydrostatic test case}

Still water situations in presence of variable bed and channel shape are a challenging problem for advection schemes. In this case, the equations in quasi-conservative form (10) reduce to:

$$
\frac{\partial z_{s}}{\partial x}=0
$$

that is, the free surface level is uniform. Advection schemes are not always able to keep the static equilibrium at the discrete level. A test case proposed by [20] has been selected. It is a channel rectangular in cross section with variable width and bed level as Fig. 4 shows. A Manning coefficient $n=0.015$ is assumed. The evolution in time of an initial uniform $12 m$ free surface level of motionless water will be studied during $200 \mathrm{~s}$ in a 150 cell grid. Fig. 4 shows that both, the unified discretization and the pointwise discretization of the fiction term, in the scheme considered in section 4 preserves a perfect hydrostatic equilibrium. This due to the fact that the schemes are perfectly balanced at the discrete level and do not produce any spurious discharge in this case of still water. 


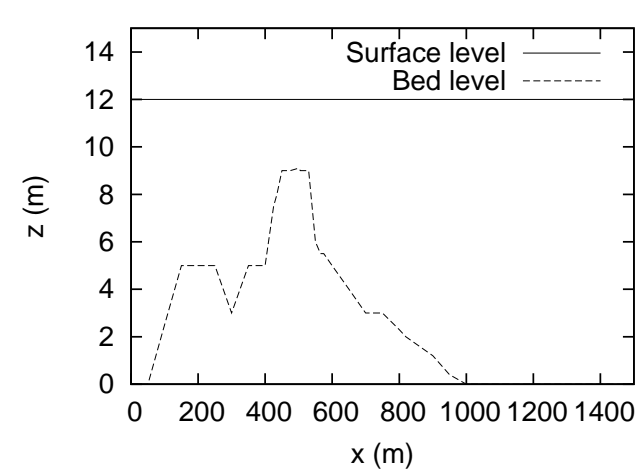

(a)

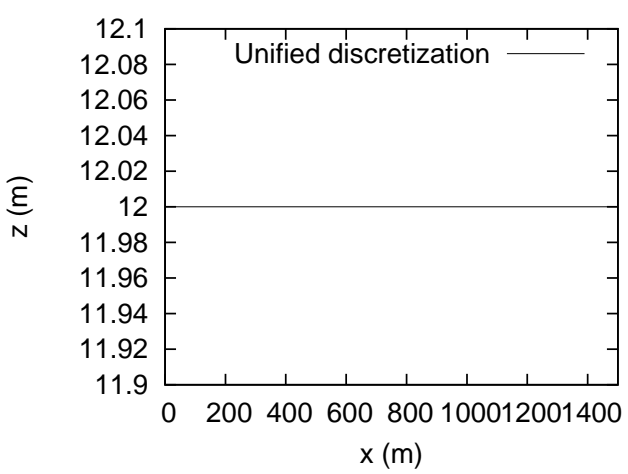

(c)

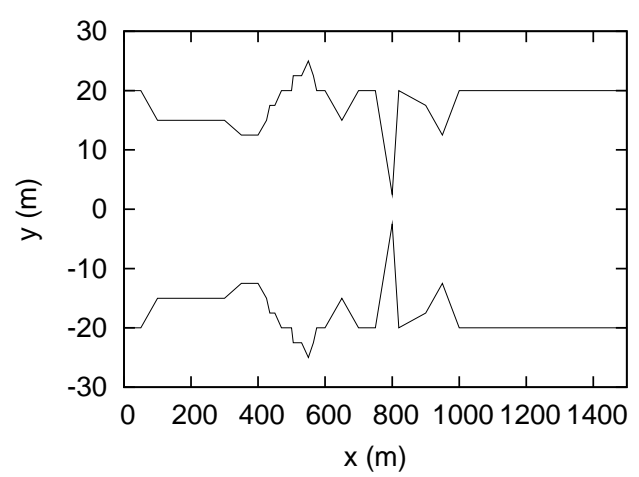

(b)

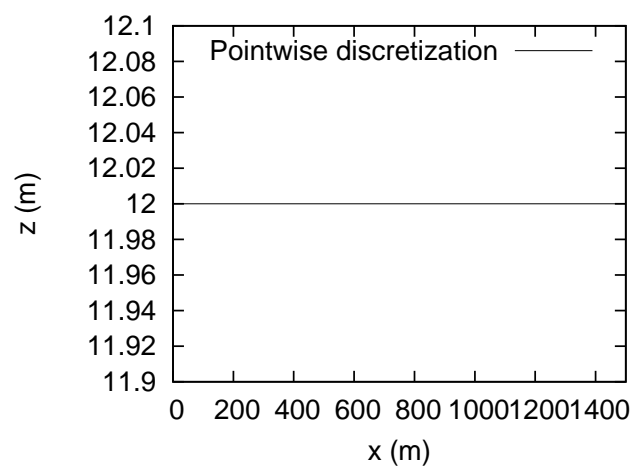

(d)

Figure 4. Hydrostatic test case: water surface and bed longitudinal profiles (a) initial, final using (c) unified, (d) pointwise discretization of the friction term. (b) plant view.

\subsection{MacDonald's test case}

MacDonald [21, 22] proposed a series of steady open channel flow test cases with analytical solution. In all of them sloping bed and friction force are considered. The steady Saint-Venant equations (1), with $\beta=1$, are:

$$
\begin{gathered}
Q=\text { const. } \\
\frac{\partial}{\partial x}\left(\frac{Q^{2}}{A}+g I_{1}\right)=g\left(I_{2}+A S_{0}\right)-T
\end{gathered}
$$


Given a constant discharge and an analytical water depth function, the steady solutions can be found from the momentum equation:

$$
S_{0}=\frac{1}{g A}\left[T-g I_{2}+\frac{\partial}{\partial x}\left(\frac{Q^{2}}{A}+g I_{1}\right)\right]
$$

where $T$ is modelled via (12). Then, the channel bed form function can be obtained.

One of these test cases has been chosen to show the influence of the discretization of the friction term on the quality of the steady state solution. A subcritical flow in a channel of rectangular cross section $10 \mathrm{~m}$ wide and $150 \mathrm{~m}$ long is assumed. The steady discharge is $20 \mathrm{~m}^{3} / \mathrm{s}$ and the bed material is characterised by a friction Gauckler-Manning coefficient $0.03 \mathrm{sm}^{-1 / 3}$. The water depth is defined by the function:

$$
h=0.8+0.25 \exp \left[-\frac{135}{4}\left(\frac{x-75}{150}\right)^{2}\right]
$$

Dry initial conditions and a time of $800 \mathrm{~s}$ are used to reach convergence to the steady state. Figure 5 shows the analytical water surface and bed level longitudinal profiles, and the numerical results obtained using the second order TVD scheme on a 50 cell grid and both the unified and pointwise discretizations of the friction term after convergence to steady state. It can be seen that only the unified method is able to provide a right balance and a perfect conservation in the steady discharge.

\subsection{Experiments on an impervious irrigation border}

The experimental data from [23] were used to illustrate the improvement of the proposed limitation of the friction term in cases of unsteady flow in conditions of high relative roughness. In that experiment a free-draining irrigation border $200 m$ long and $2 m$ wide, with a slope of $S_{0}=0.000671$ was constructed and covered with plastic film. A fine layer gravel (with $d_{50}$ of approximately $10 \mathrm{~mm}$ ) was added on top of the plastic film. A steady flow case was measured 


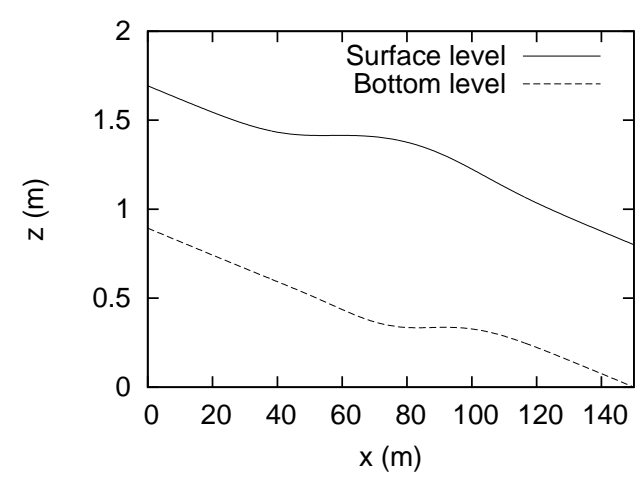

(a)

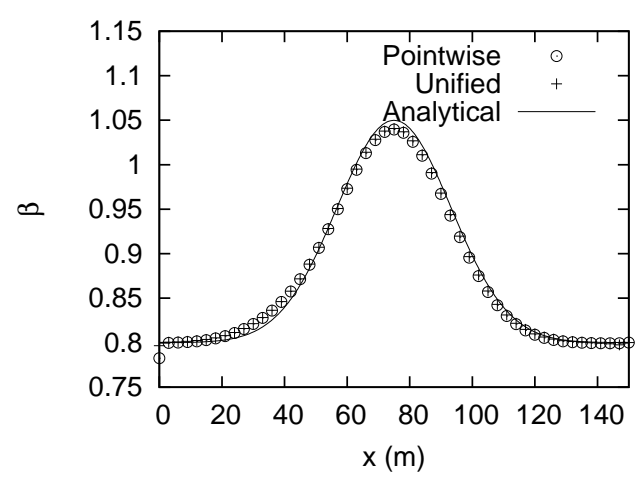

(b)

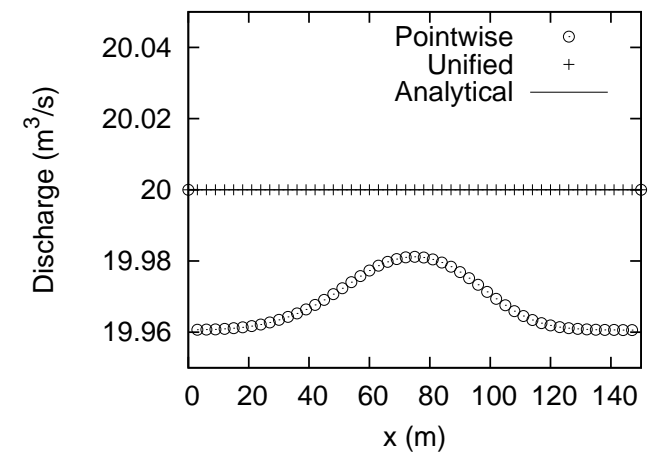

(c)

Figure 5. (a) Analytical surface and bottom levels, (b) depth and (c) discharge with different treatments of the friction term in the MacDonald test case.

for a discharge of $Q=0.010 \mathrm{~m}^{3} / \mathrm{s}$. Two unsteady experiments of water flow advancing over the dry border bed were performed using inlet discharges of $Q=0.0047 \mathrm{~m}^{3} / \mathrm{s}$ and $Q=0.0117 \mathrm{~m}^{3} / \mathrm{s}$. For the numerical simulation of the unsteady flow a second order TVD scheme with "Superbee" flux limiter with unified and implicit $(\theta=0.5)$ discretization of the friction term has been used.

The roughness coefficient is estimated assuming normal flow $\left(S_{0} \approx S_{f}\right)$ conditions at steady state using the experimental steady flow depth of $H=50 \mathrm{~mm}$. Then, from (13) or from (21):

$$
n \approx \frac{\sqrt{S_{0}} H^{2 / 3}}{U}, \quad \epsilon \approx \frac{g S_{0}}{(b+1)^{2} U^{2}}\left(\frac{H^{b+(1 / 2)}}{l^{b}}-\frac{l}{\sqrt{H}}\right)^{2}
$$


Considering the granulometry of the gravel, a characteristic roughness length of $l=d_{50}=$ $10 \mathrm{~mm}$ is used and $b=0.25$ typical in gravel bed rivers [6], a value of $\epsilon=0.12$ has been adjusted. In all cases, this length is used as the water depth threshold value for water movement.

Figure 6 is a plot of the discharge longitudinal profiles for $Q=0.0117 \mathrm{~m}^{3} / \mathrm{s}$ and $t=2400 \mathrm{~s}$ simulated with different mesh cell sizes with and without limiting source term. It shows that when the friction force is not limited, the numerical solution becomes more and more unstable as the the grid size is increased despite the implicit discretization of the source term. With the proposed bounding limit for the discrete friction force, the numerical scheme is not only stable for any grid size (under the CFL condition) but is also able to produce numerical solutions on coarser grids of the same quality as those obtained in finer grids with the non-limited method.

This fact is also noticeable in figure 7 where the time advance of the wetting front is plotted for inlet discharges $Q=0.0047 \mathrm{~m}^{3} / \mathrm{s}$ and $Q=0.0117 \mathrm{~m}^{3} / \mathrm{s}$ as computed on different meshes with and without limiting friction term. The results show that the numerical advance is independent of the grid size when the limitation over the friction term is applied. They also show that the same accuracy is reached with this technique on a coarse grid and by the non-limited technique on a much finer grid.

Finally, figure 8 compares the advancing front computed using the Manning and power law friction models. In both cases the limiting technique of the discrete friction force is applied. Both models predict reasonably well the advance with a slight tendency to overpredict the advancing speed. A somehow better prediction is provided by the power law model. 

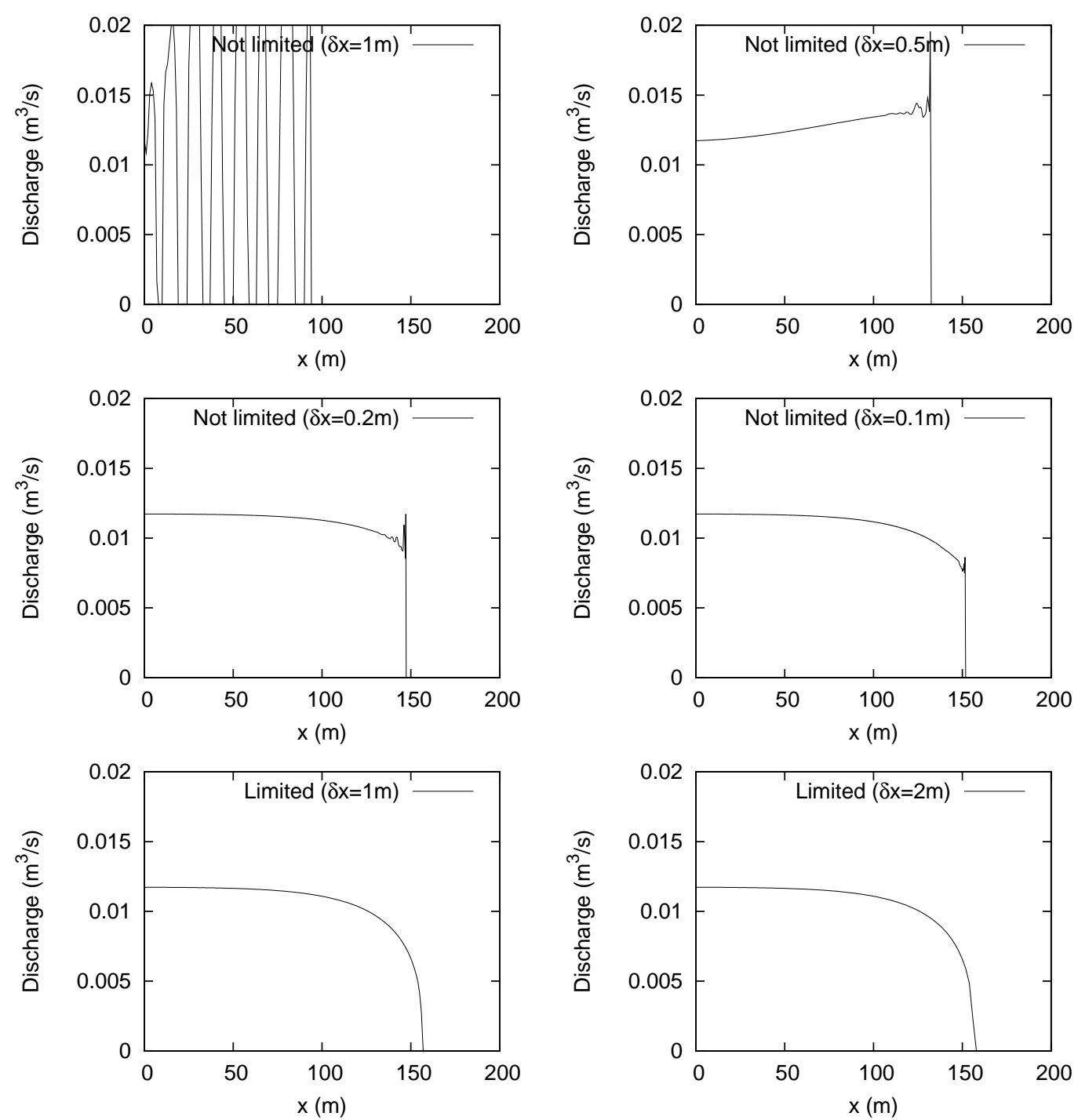

Figure 6. Discharge longitudinal profiles for $Q=0.0117 \mathrm{~m}^{3} / \mathrm{s}$ and $t=2400 \mathrm{~s}$ simulated with different mesh cell sizes with and without limiting friction term. Power law friction model with $b=0.3$,

$$
l=10 \mathrm{~mm} \text { and } \epsilon=0.12 \text { was used. }
$$

\subsection{Neila River}

Neila River flows through a mountain region of central Spain. It is highly irregular in shape and steep (with average slope around $20 \%$ in some reaches) as it can be seen in figure 9 . The 


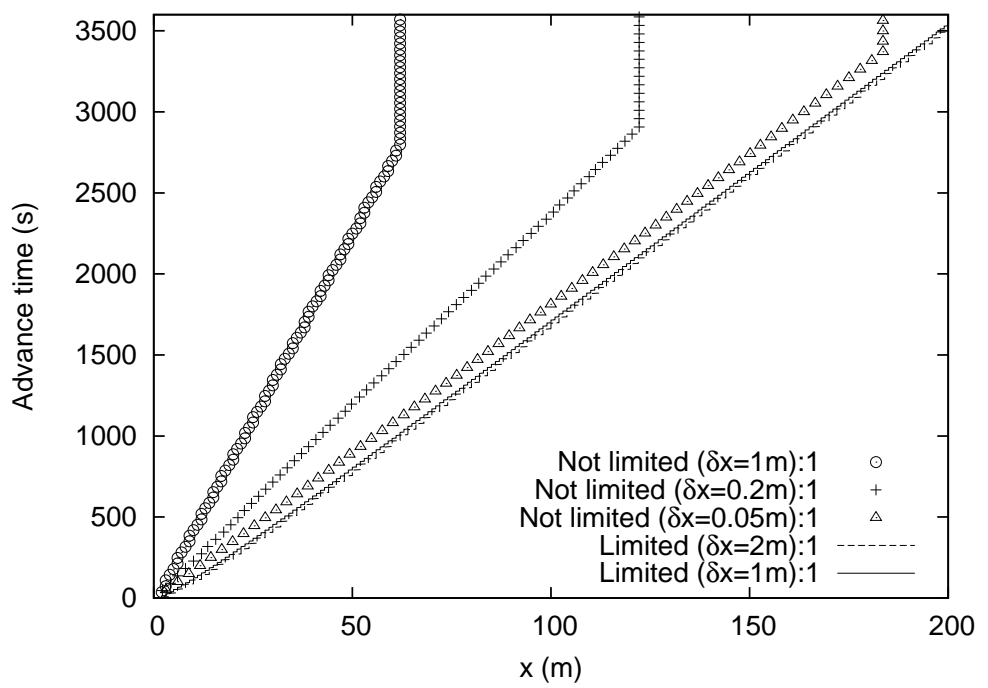

(a)

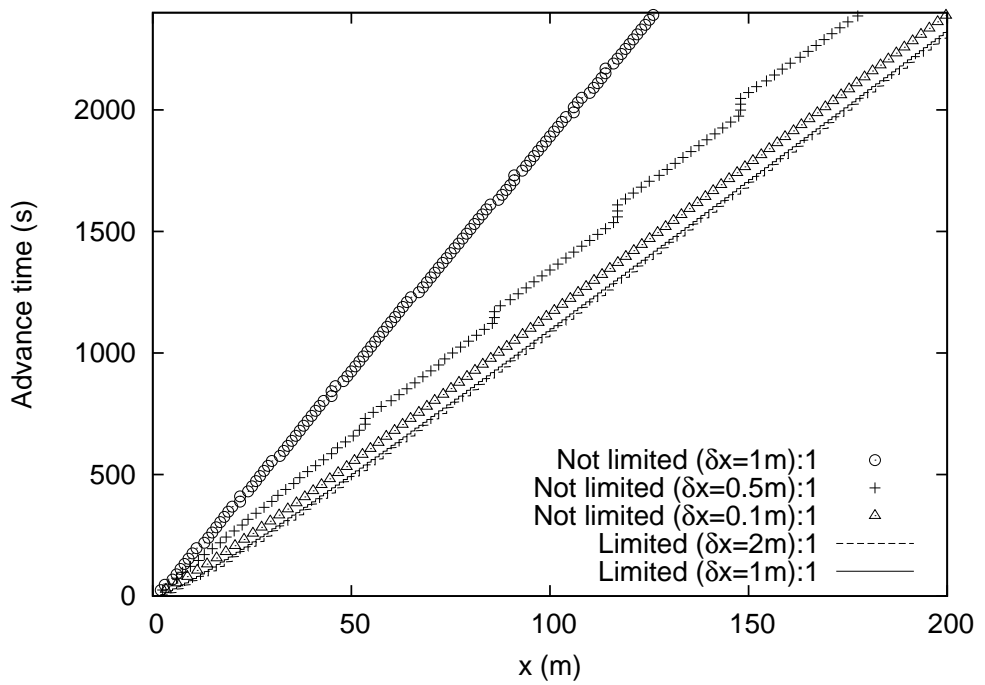

(b)

Figure 7. Advance curves for (a) $Q=0.0047 \mathrm{~m}^{3} / \mathrm{s}$, (b) $Q=0.0117 \mathrm{~m}^{3} / \mathrm{s}$ and different cell sizes with and without limiting friction term. Power law friction model with $b=0.3, l=10 \mathrm{~mm}$ and $\epsilon=0.12$ was used. 


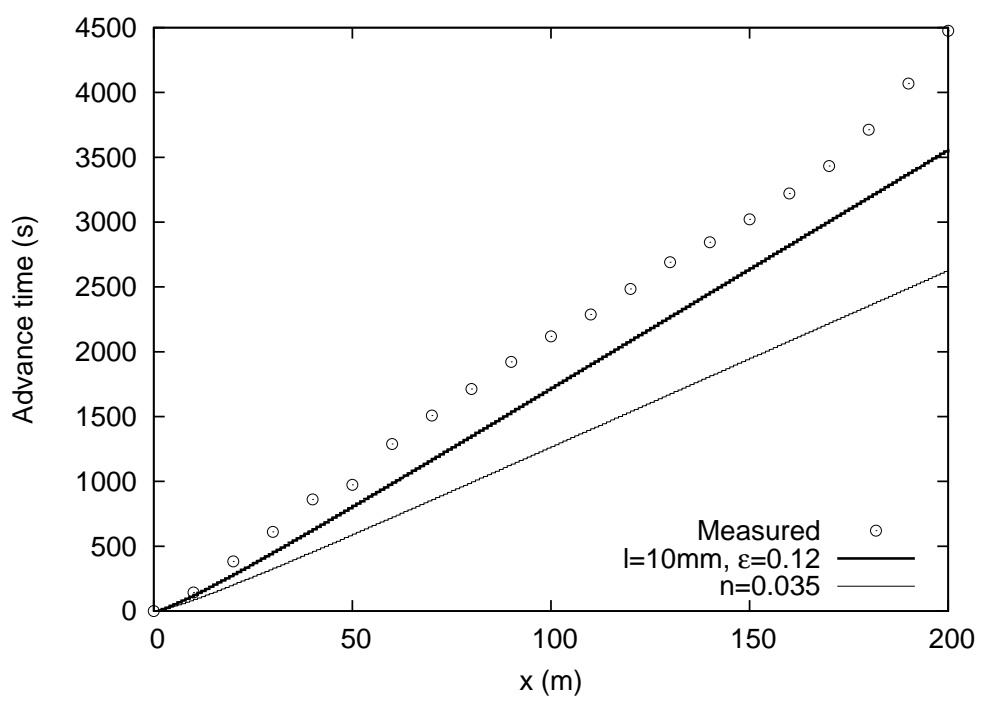

(a)

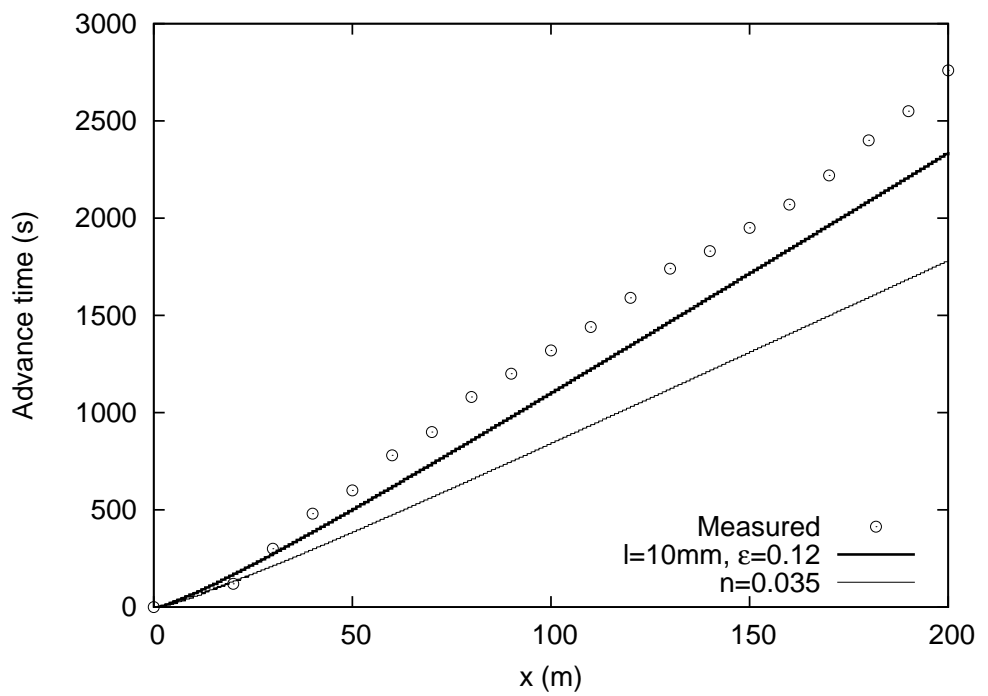

(b)

Figure 8. Advance curves for (a) $Q=0.0047 \mathrm{~m}^{3} / \mathrm{s}$ and (b) $Q=0.0117 \mathrm{~m}^{3} / \mathrm{s}$ measured and simulated with $\delta x=1 m$ and limiting friction term for different friction models. 
base flow is very low (around $1 \mathrm{~m}^{3} / \mathrm{s}$ ) and the stony but vegetated river bed is associated to a high friction factor $n=0.05 \mathrm{sm}^{-1 / 3}$. A reach of $18 \mathrm{Km}$ has been characterized by means of 48 cross sections. The physical conditions of steep slope, low base discharge, high roughness and many transcritical points make this river a challenging test case for the numerical schemes to be able to produce a stable solution. The simulations are made with the second order TVD scheme with an implicit $(\theta=0.5)$ unified discretization of the friction term. A first run was performed to reach base flow steady state conditions starting from dry bed initial conditions.

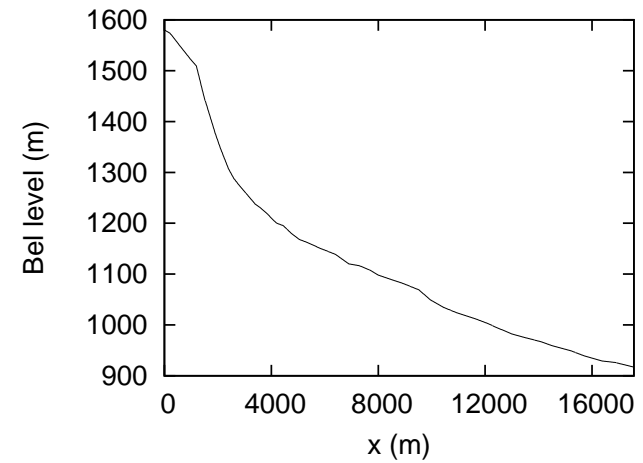

(a)

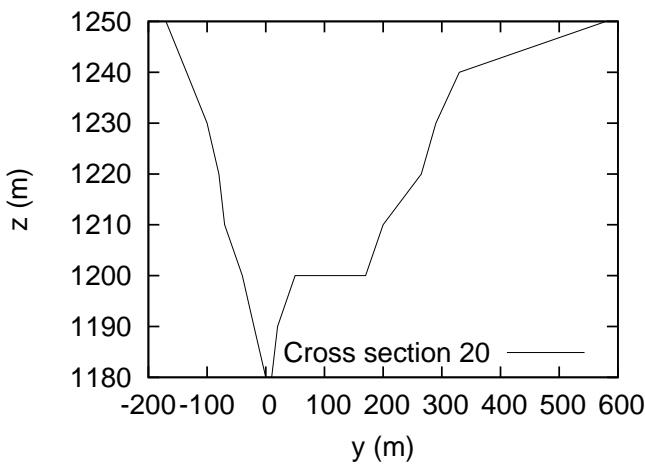

(c)

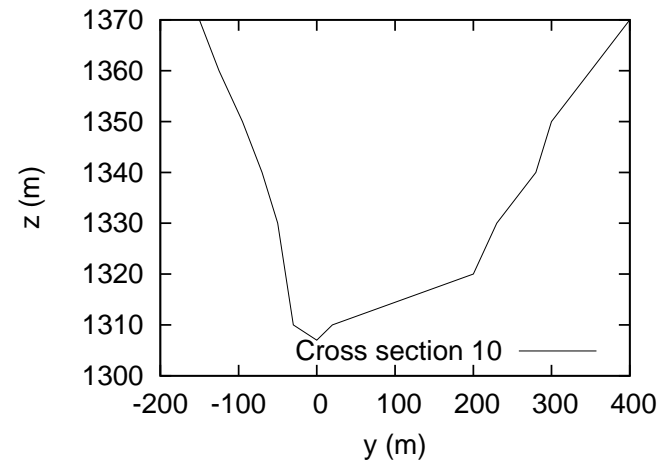

(b)

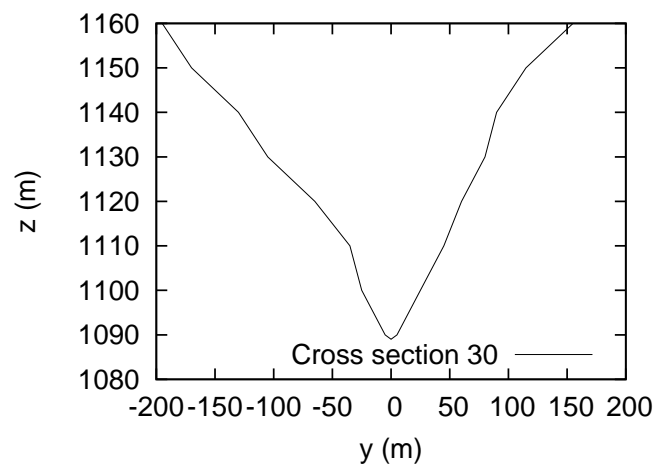

(d)

Figure 9. Bed slope and three typical cross sections of the Neila River. 
Several spatial grid and time step sizes have been combined in order to estimate their influence in the numerical results during this first run. In all cases, the following water depth threshold value, derived from (40) was imposed:

$$
h \leq\left(\frac{g n^{2} \delta x}{2}\right)^{\frac{3}{4}} \Rightarrow Q=0
$$

This condition is necessary to stabilise the solution since it avoids unrealistic growing tendencies in the discrete friction terms. It stops water at depth values less than the limit imposed leading, for coarse grids, to some kind of pulses in the flow during the transient calculation that spoil the quality of the solution.

The water depth in the base flow steady profile can be as low as $4 \mathrm{~cm}$ in some parts producing, from the stability condition (40), that $\delta x \leq 1.1 m$, is necessary to ensure smooth, continuous and steady discharge result when no limiting the friction term. As the grid size is increased above that limit, and despite the implicit discretization of the friction term, the numerical solutions become eventually unstable. The traditional remedy of trying to reduce the instabilities by decreasing the time step size does not work since, using in $\delta x=2 m$ the time step given by $\mathrm{CFL}=0.25$ or $\mathrm{CFL}=0.02$ in the grid $\delta x=10 \mathrm{~m}$, does not improve the stability of the solution due to the inadequacy of the space discretization. However, applying the proposed limitation over the numerical friction force the scheme remains stable with larger grid sizes.

In a second run, the hydrograph deduced from a hypothetical failure of a dam located upstream of the river reach was used as inlet boundary condition over the initial conditions given by the base flow steady state. In this simulation, the friction force limiting technique was used as well as $\delta x=15 \mathrm{~m}$ and $\mathrm{CFL}=0.9$. Figure 11 shows the inlet hydrograph and the longitudinal profiles of discharge, water depth, Froude number and $\beta$ at different times. The various sub/supercritical transitions are noticeable. The estimate of $\beta$ according to (17) shows 

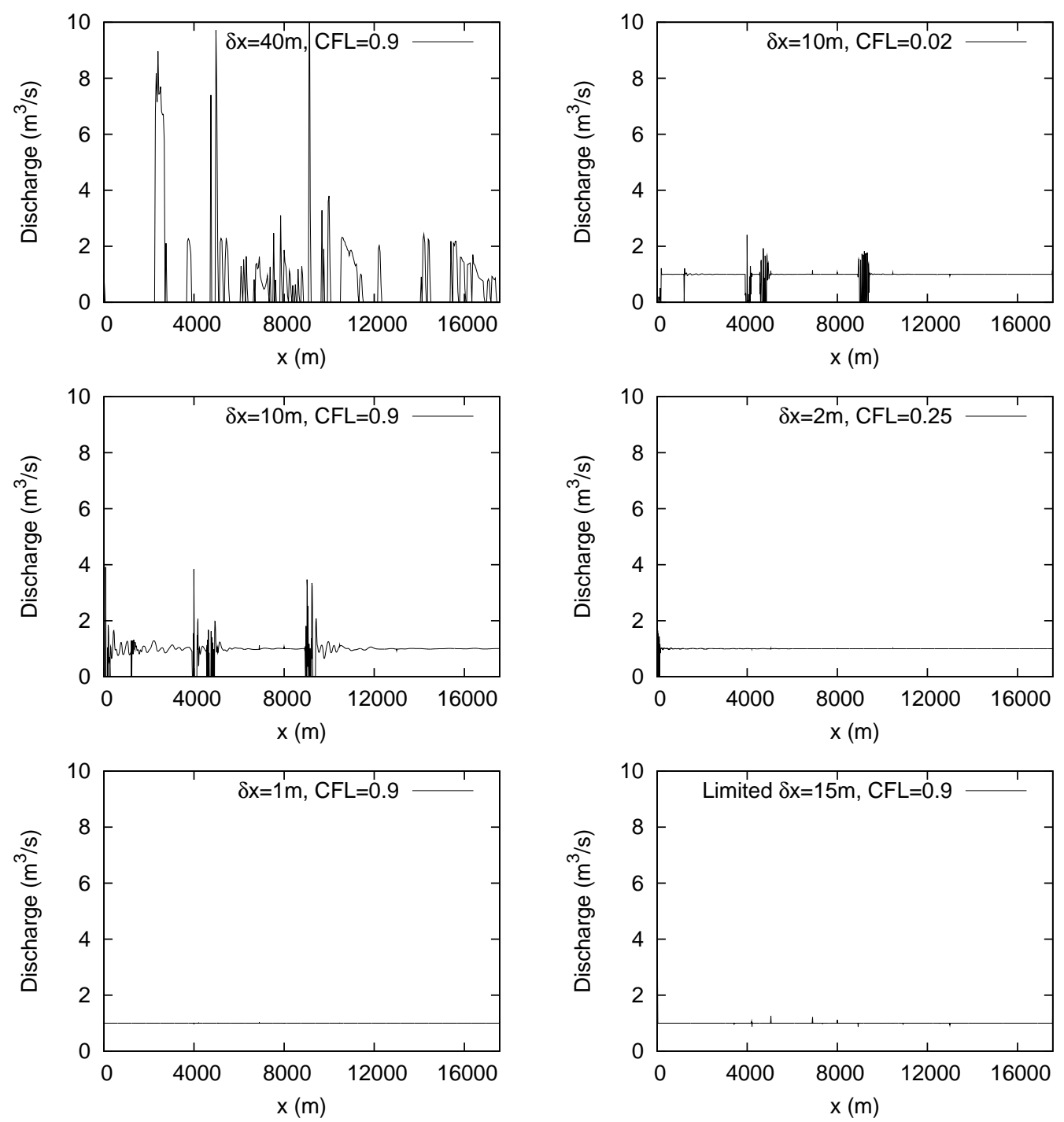

Figure 10. Steady state longitudinal profile of discharge for Neila River using different time step and cell mesh sizes.

the values reached by this parameter in natural rivers, as indicated in $[25,6]$. 


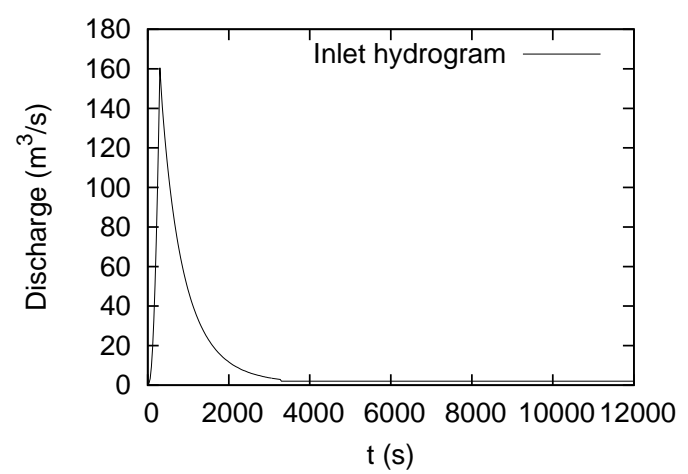

(a)

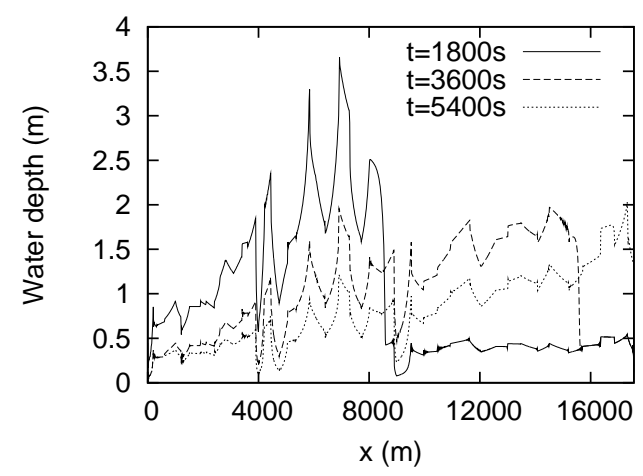

(c)

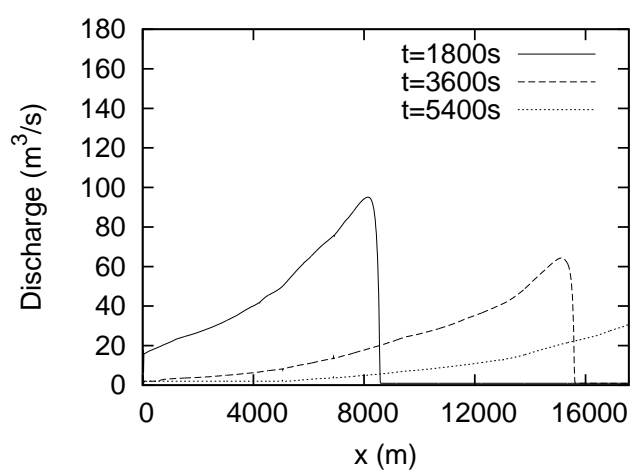

(b)

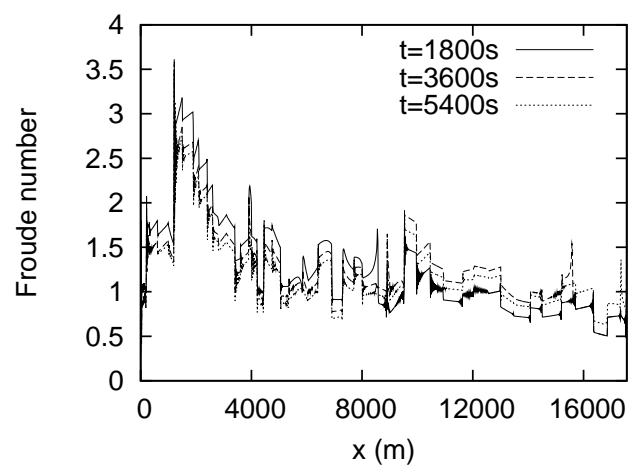

(d)

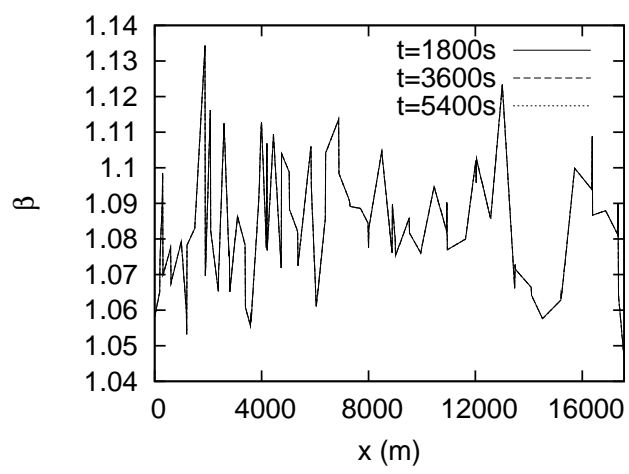

(e)

Figure 11. (a) Inlet hydrograph, (b) discharge, (c) water depth, (d) Froude number and (e) $\beta$ coefficient simulated in Neila River at different times. 


\subsection{Tsunami runup onto a complex three-dimensional beach}

This test case has been taken from a benchmark problem of the Third International Workshop on Long Wave Runup Models. It is an 1/400 scale laboratory experiment of the Monai runup (Okushiri Island, Japan) using a large-scale tank (205 m long, $6 \mathrm{~m}$ deep, $3.4 \mathrm{~m}$ wide) at Central Research Institute for Electric Power Industry (CRIEPI) in Abiko, Japan. A benchmark test case was defined focusing on a region near the shoreline where experimental data were measured. The detailed description as well as the experimental data are available from [24]. This test case description does not provide any friction information. According to the reported bed material we have use a Gauckler-Manning number of $n=0.015 \mathrm{sm}^{-1 / 3}$. For the simulation, the (16) and (17) models were used in a cell grid size of $0.014 m$. It is emphasised that the problem simulates the runup in the reduced domain $5.4 m$ long and $3.4 m$ wide. Figures 12 and 13 shown below represent the bathymetry used in the reduced region and the location of the gauging points. The incident wave from offshore, at the water depth $h=13.5 \mathrm{~cm}$ was introduced in our 1D model through the inlet boundary. In the laboratory model the other three boundaries were reflective vertical sidewalls and so has been considered in our model.

Figure 14 show the time evolution of the water surface level at the inlet (a) (upstream boundary condition), and the result from the simulation at $x=4.521 \mathrm{~m}$ (b) together with the measurements provided at three gauging points with the same $x$ and different $y$ coordinate position. The plot shows that, despite the complexity of the bottom surface and the twodimensional character of the example, the one-dimensional model provides fairly well the wave arrival time, shape and amplitude. 


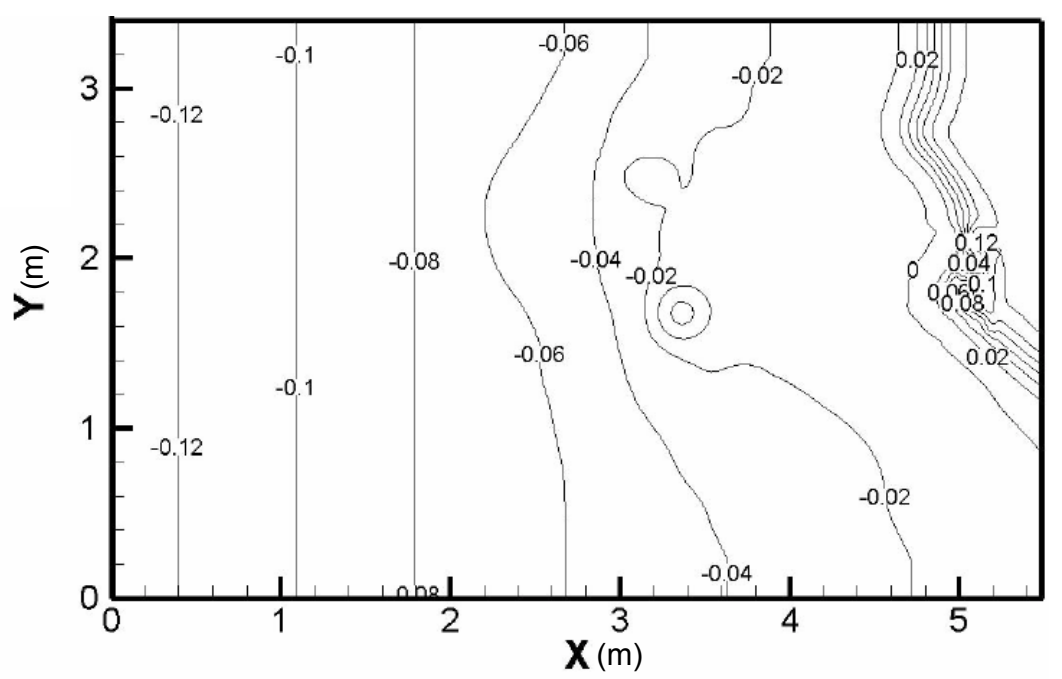

Figure 12. Plant view of the domain and bed level contour lines

\section{CONCLUSIONS}

Two main options for the discretization of the friction term in the one-dimensional shallow water equations have been detailed, analysed and compared. One of them is the pointwise discretization, separate and independent of the technique used for the rest of the terms in the dynamic equation and the other is the unified discretization in which all the terms are treated in a similar form.

The comparison of the two techniques in cases of steady flow show that the pointwise discretization, although stable when fully implicit, is unable to provide a good balance among 


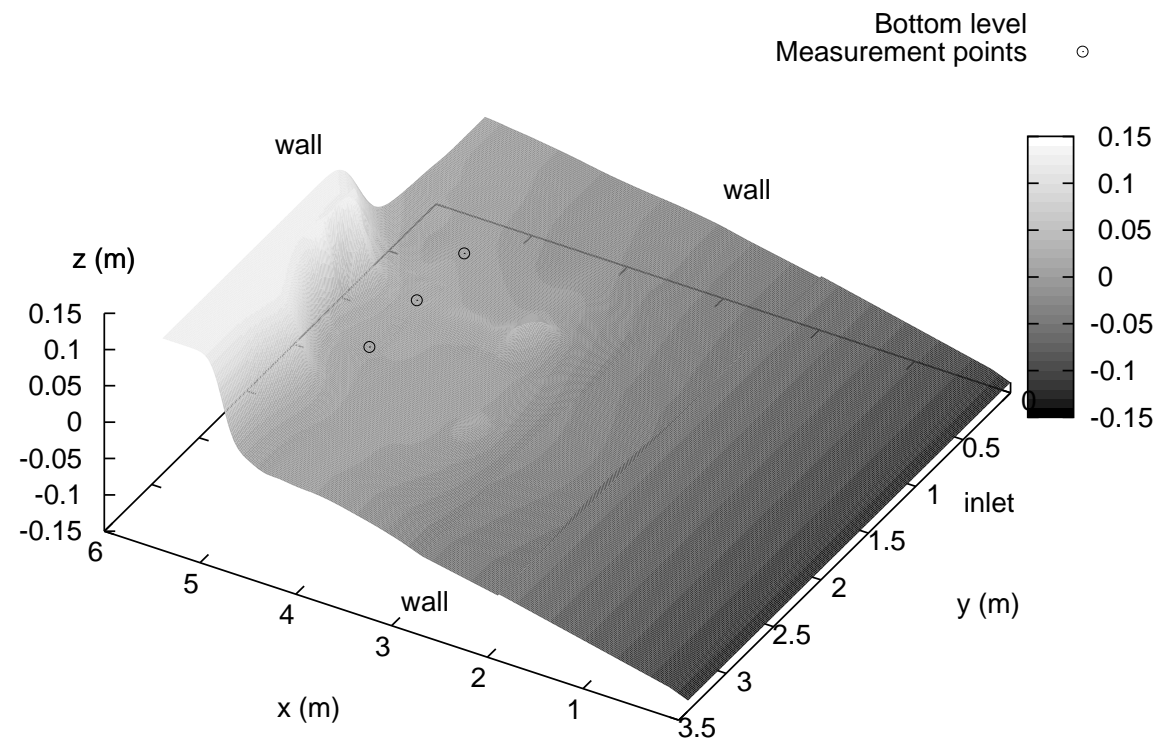

Figure 13. Bathymetry and measurement points. Note that the vertical scale is distorted.

the terms of the equation hence producing bad quality steady states.

Furthermore, a form of limiting the value of the discrete friction force is proposed in order to prevent that the numerical model produces values exceeding the maximum friction force physically allowed. This limitation improves the quality of unsteady solutions in wet/dry fronts and guarantees the numerical stability in cases with dominant friction terms.

\section{REFERENCES}

1. Darcy HPG (1858). Recherches expérimentales relatives aux mouvements de l'eau dans les tuyaux. Mémoires Présentées à l'Académie des Sciences, Paris.

2. Gauckler PG (1867). Études théoriques et pratiques sur l'écoulement et le mouvement des eaux. Comptes Rendues de l'Académie des Sciences, Paris. 


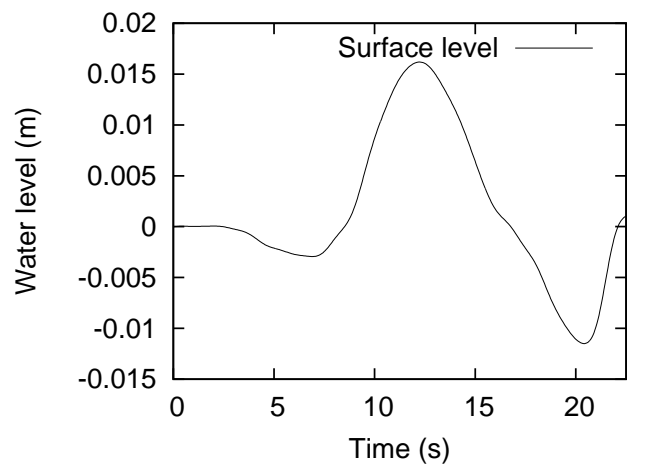

(a)

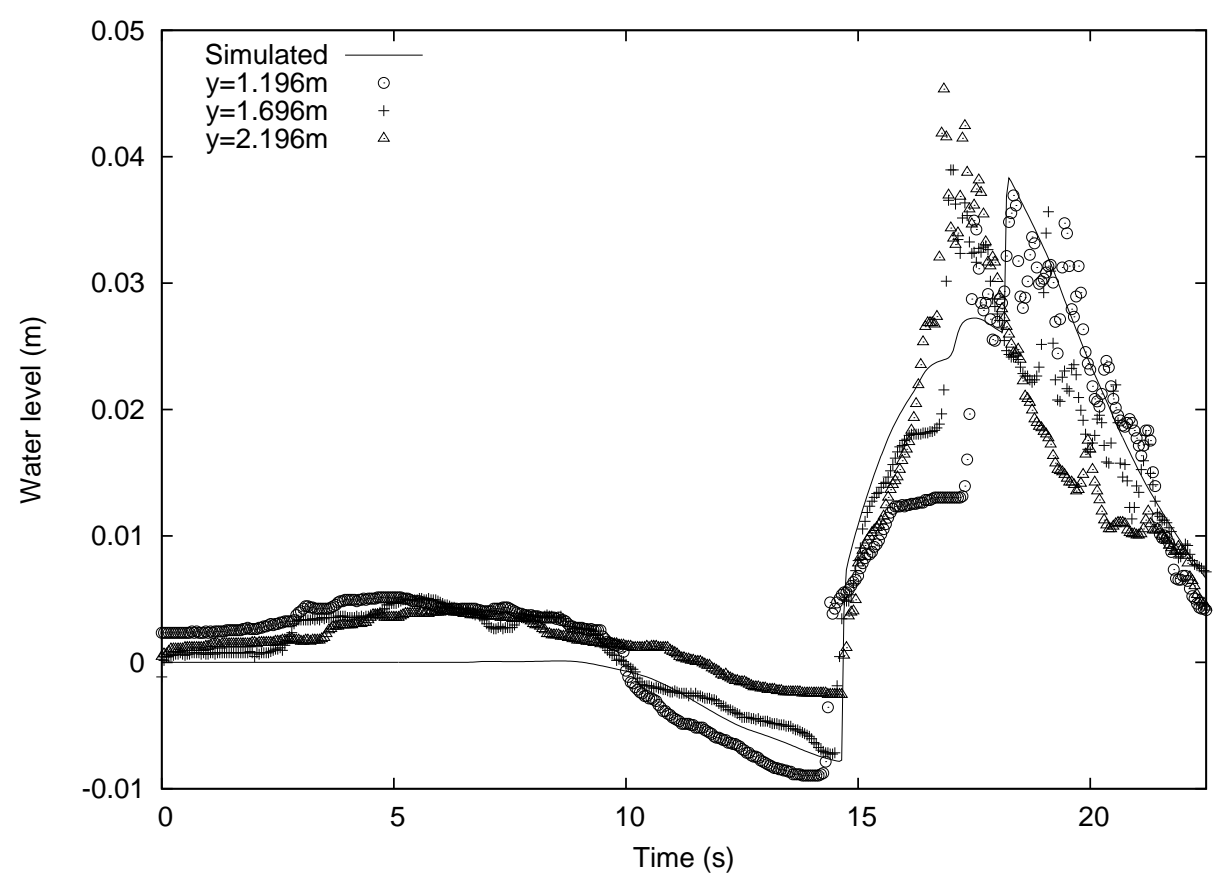

(b)

Figure 14. Time evolution of the water surface level (a) at the inlet section, (b) at $x=4.521 \mathrm{~m}$ as simulated and measured at three points with different $y$ position.

3. Manning R (1890). On the flow of water in open channels and pipes. Institution of Civil Engineers of Ireland.

4. Chow VT (1959). Open channel hydraulics. McGraw-Hill, New York. 
5. Smart GM (1999). "Turbulent velocity profiles and boundary shear in gravel-bed rivers". ASCE Journal of Hydraulic Engineering, 125(2), 106-116.

6. Burguete J, García-Navarro P, Murillo J, García-Palacín I. Analysis of the friction term in the onedimensional shallow-water model. ASCE Journal of Hydraulic Engineering, in press.

7. Huang J, Song CCS (1985). "Stability of dynamic flood routing schemes". ASCE Journal of Hydraulic Engineering, 111(12), 1497-1505.

8. García-Navarro P, Fras A, Villanueva I (1999). "Dam-break flow simulation: some results for onedimensional models of real cases". Journal of Hydrology, 216, 227-247.

9. Burguete J, García-Navarro P (2001). "Efficient construction of high-resolution TVD conservative schemes for equations with source terms: application to shallow-water flows". International Journal for Numerical Methods in Fluids, 37(2), 209-248.

10. Burguete J, García-Navarro P (2004). "Improving simple explicit methods for unsteady open channel and river flow". International Journal for Numerical Methods in Fluids, 45(2), 125-156.

11. Bermúdez A, Vázquez-Cendón ME (1994). "Upwind methods for hyperbolic conservation laws with source terms". Computers and Fluids, 23(8), 1049-1071.

12. García Navarro P, Vázquez Cendón ME (2000). "Some considerations and improvements on the performance of Roe's schemes for 1D irregular geometries on numerical treatment of the source terms in the shallow water equations". Computers and Fluids, 126(1), 26-40.

13. Courant R, Friedrichs KO, Lewy H (1928). "Über die partiellen Differenzengleichungen der mathematischen Physik". Mathematische Annalen, 100(1), 32-74.

14. Saint-Venant AJCB de (1871). Théorie de mouvement non-permanent des eaux avec application aux crues de rivières et á l'introduction des marées dans leur lit. Comptes Rendues de l'Académie des Sciences, Paris.

15. Burguete J, García-Navarro P (2004). "Implicit schemes with large time step for non-linear equations: application to river flow hydraulics". International Journal for Numerical Methods in Fluids, 46(6), 607636

16. Burguete J, García-Navarro P, Murillo J. Preserving bounded and conservative solutions of transport in 1D shallow-water flow with upwind numerical schemes: Application to fertigation and solute transport in rivers. Submited to International Journal for Numerical Methods in Fluids.

17. Roe PL (1984). Generalized formulation of TVD Lax-Wendroff schemes. ICASE Report 84-53, NASA CR-172478, NASA Langley Research Center.

18. Albada GD van, Leer B van, Roberts WW (1982). "A comparative study of computational methods in 
cosmic gas dynamics". Astron. Astrophysics, 108, 76-84.

19. Leer B van (1974). "Towards the ultimate conservative difference scheme II. Monotonicity and conservation combined in a second order scheme". Journal of Computational Physics, 14, 361-370.

20. Goutal N, Maurel F (1997). Proceedings of the 2nd workshop on dam-break wave simulation. HE43/97/016/B, Direction des Études et Recherches, EDF.

21. MacDonald I (1996). Analysis and computation of steady open channel flow. PhD thesis, University of Reading.

22. MacDonald I, Baines MJ, Nichols NK, Samuels PG (1997). "Analytical benchmark solutions for openchannel flows". ASCE Journal of Hydraulic Engineering, 123(11), 1041-1045.

23. García-Navarro P, Playán E, Zapata N (2000). "Solute transport modeling in overland flow applied to fertigation". ASCE Journal of Irrigation and Drainage Engineering, 126(1), 33-41.

24. Matsuyama M. Central Research Institute for Electric Power Industry, Abiko, Japan. "The third international workshop on long-wave runup models" . (2004). Wrigley Marine Science Center, Catalina Island, California. <http://www.cee.cornell.edu/longwave/index.cfm?page=benchmark\&problem=2> (downloaded Apr. 12, 2007).

25. Cao Z, Meng J, Pender G, Wallis S (2006). "Flow resistance and momentum flux in compound open channels". ASCE Journal of Hydraulic Engineering, 132(12), 1272-1282. 